\title{
Aryl hydrocarbon receptor activation by aminoflavone: New molecular target for renal cancer treatment
}

\author{
MARIANA A. CALLERO ${ }^{1 *}$, GUADALUPE V. SUÁREZ ${ }^{1 *}$, GABRIELA LUZZANI $^{1}$, \\ BORIS ITKIN ${ }^{2}$, BINH NGUYEN $^{3}$ and ANDREA I. LOAIZA-PEREZ ${ }^{1}$ \\ ${ }^{1}$ Research Area, Institute of Oncology ‘Ángel H. Roffo’, University of Buenos Aires, Ciudad de Buenos Aires; \\ '`J. Fernández’ General Hospital, Buenos Aires, Argentina; ${ }^{3}$ Tigris Pharmaceuticals Inc., Bonita Springs, FL, USA
}

Received December 10, 2011; Accepted January 27, 2012

DOI: 10.3892/ijo.2012.1427

\begin{abstract}
Aminoflavone (AF; NSC 686288, AFP464, NSC710464) is a new anticancer drug that has recently entered phase II clinical trials. It has demonstrated antiproliferative effects in MCF-7 human breast cancer cells mediated by the aryl hydrocarbon receptor (AhR). AF also exhibits noteworthy evidence of antitumor activity in vitro and in vivo against neoplastic cells of renal origin. AF treatment of sensitive renal cells, in contrast to resistant cells, promotes the induction of CYP1A1, the covalent binding of AF-reactive intermediates and apoptosis. Based on this evidence, the aim of this study was to evaluate the role of $\mathrm{AhR}$, the main transcriptional regulator of CYP1A1, in the antiproliferative effects of AF in human renal cancer cells. AF-cytoxicity in human renal cell lines and a renal cancer cell strain was assessed by MTS assay in the presence or absence of an Ahr inhibitor. Drug-induced AhR nuclear translocation was evaluated by western blotting of AhR in cytosolic and nuclear fractions and by measuring xenobiotic response element-driven luciferase activity. Apoptosis induced by the drug was evaluated by 4,6-diamidino-2-phenylindole and acridine orange/ethidium bromide staining and by measuring phosphorylated P53 (p-P53) and P21 levels, caspase 3 activation and poly(ADP-ribose) polymerase cleavage. AF inhibited cell growth in a dose-dependent manner in TK-10, Caki-1, SN12-C and A498 human renal cells but not in ACHN cells. The antiproliferative effect of AF was abrogated by preincubation of TK-10, Caki-1 and SN12-C cells with the AhR antagonist, $\alpha$-naphthoflavone. AF treatment also induced apoptosis in TK-10, Caki-1 and SN12-C cells, which was not
\end{abstract}

Correspondence to: Dr Andrea I. Loaiza-Perez, Research Area, Institute of Oncology 'Ángel H. Roffo', University of Buenos Aires, Avenue San Martín 5481, C1417DTB Ciudad de Buenos Aires, Argentina

E-mail: loaizaa2012@gmail.com

${ }^{*}$ Contributed equally

Key words: aryl hydrocarbon receptor, cytochrome P450 1A1, renal cancer, apoptosis observed in ACHN cells. AF induced time-dependent AhR nuclear translocation and AhR transcriptional activity in sensitive renal cancer cell lines. A renal cell strain derived from a human papillary tumor also showed sensitivity to AF, as well as AhR pathway activation and drug-induced apoptosis. AhR translocation could be included as a marker of sensitivity to AF in sensitive renal tumor cells of different histological origin, in ongoing phase II clinical trials.

\section{Introduction}

Renal cell carcinoma represents 5\% of epithelial neoplasias and its incidence is increasing constantly over the last 30 years (1). Histologically, the majority of cases (70-85\%) are clear cell carcinomas (1-4), whereas other less frequent subtypes are papillary cell carcinomas (10-15\%) (4). Metastatic renal carcinoma is resistant to conventional chemotherapy and is almost always incurable (2). Over the last few years, phase II and III clinical studies with new molecular anti-angiogenic therapies, such as multikinase inhibitors, monoclonal antibodies against vascular endothelial growth factor in combination with interferon- $\alpha$, as well as m-TOR inhibitors, have shown statistically significant benefits in terms of free progression and global survival. However, the average of survival without progression rarely exceeds one year (1-4). For this reason, the development of new effective agents with alternative mechanisms of action is of great importance.

Aminoflavone [AF, 4H-1-benzopyran-4-one, -amino-2(4amino-3-fluorophenyl)-6,8-difluoro-7-methyl, NSC686288; AFP464, NSC710464] (Fig. 1) is a novel antitumor candidate agent, that is currently undergoing phase II clinical trials. This compound has a distinct COMPARE pattern of cytotoxicity in the NCI60 cell line and has a potent antiproliferative activity on human breast and renal tumor xenografts $(5,6)$.

In a previous study, we demonstrated that the antiproliferative effect of AF on MCF-7 breast tumor cells is mediated by the aryl hydrocarbon receptor (AhR), which upon dimerization with hypoxia-inducible factor $1 \beta$ (HIF-1 $\beta$ )/AhR nuclear translocator activates transcription by binding to the xenobiotic response element (XRE) in the promoters of target genes, including cytochrome P450 (CYP)1A1 (5). The AF pro-drug induces its own metabolism through CYP1A1 activation. This is a unique aspect of its anticancer action which differs from 
other available anticancer agents, although the exact mechanism by which AF exerts its anticancer activity has not been fully elucidated.

AF activity in sensitive breast tumors has been linked to the presence of cytoplasmic AhR, the translocation of the AhR-AF complex to the nucleus followed by induction CYP1A1 (5), activation of sulfotransferase 1A1 (7) oxidative stress (8) and DNA damage caused by metabolites (9). The latter is exemplified by the occurrence of $\gamma-\mathrm{H} 2 \mathrm{AX}$ phosphorylation consistent with the induction of DNA single-strand breaks and DNA-protein crosslinks (9). Breast cancer resistant cells did not show induction of CYP1A1 expression by AF, which seems to be essential for its antiproliferative activity. ER-positive $\left(\mathrm{ER}^{+}\right)$breast cancer cells are generally sensitive to AF. It has been reported that $\mathrm{ER}^{+}$ cells which became resistant to tamoxifen are still sensitive to AF (10).

In a previous study, using established human renal cancer cell lines and a series of tumor cell isolates from patients with confirmed clear cell and papillary renal disease (termed as renal cell strains), we showed that sensitivity to AF can be ascertained using several metabolic end-points, including the induction of CYP1A1 and CYP1B1 transcription, the covalent binding of AF metabolites and apoptosis (6). Furthermore, these results suggest that renal cell carcinoma of papillary origin may be more sensitive to AF than its clear cell counterpart. For papillary carcinomas that are generally unresponsive to treatment with cytokines, $\mathrm{AF}$ would be a suitable treatment (6). Based on the previous results, to examine the subcellular distribution of AhR in clear and papillary renal cells is important in order to understand more fully the mechanism of CYP1A1 mRNA induction in these AF-sensitive and AF-resistant renal tumor cells. We addressed the possibility of using the translocation of AhR as an additional susceptibility marker for the selection of patients that could be responsive if treated with AF.

We examined the molecular mechanisms involved in AhR-induced apoptosis observed after treatment with AF. The results presented in this study confirm that the activation of $\mathrm{AhR}$ in renal cancer cells plays an essential role in AF antitumor activity, mediating responsiveness and efficacy.

\section{Materials and methods}

Cell lines. The following human renal cancer cell lines were obtained from the National Cancer Institute (NCI) repository: TK-10, SN12-C, Caki-1, A498 and ACHN. The cells were cultured with $5 \% \mathrm{CO}_{2}$ in RPMI-1640 medium (Gibco) supplemented with $10 \%$ FBS (PAA). We used a cell strain obtained from a renal papillary tumor of a patient, termed as 112 . The patient was undergoing therapeutic protocols at the NCI Urologic Oncology Branch (6). The cell strain 112 was grown in Dulbecco's modified Eagle's medium containing $4.5 \mathrm{~g} / \mathrm{l} \mathrm{D}$-glucose, $250 \mu \mathrm{g} /$ $\mathrm{ml}$ Fungizone, $100 \mathrm{U} / \mathrm{ml}$ penicillin and $100 \mu \mathrm{g} / \mathrm{ml}$ streptomycin with $10 \%$ FBS. All cells were passaged weekly in their respective media.

Antiproliferative activity of AF. Renal cell lines grown in $75 \mathrm{~cm}^{2}$ $\mathrm{T}$ flasks were removed by trypsinization and seeded into 96 -well culture dishes at a concentration of 750 cells per well. For the renal cell strain 112, 4,000 cells were seeded in a 96-well plate.<smiles>Cc1c(F)c(N)c2c(=O)cc(-c3ccc(N)c(F)c3)oc2c1F</smiles>

Aminoflavone (NSC 686288)

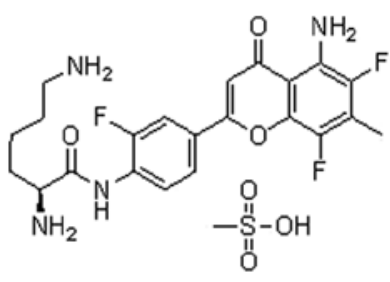

AFP464 (NSC 710464)
Figure 1. Aminoflavone structure

Cells were allowed to grow for $48 \mathrm{~h}$ at $37^{\circ} \mathrm{C}$ in a humidified atmosphere containing $5 \% \mathrm{CO}_{2}$. AF was prepared as a $100-\mathrm{mM}$ stock solution in $100 \%$ dimethylsulfoxide (DMSO). Cells were treated with $\mathrm{AF}\left(10^{-9}\right.$ to $\left.10^{-6} \mathrm{M}\right)$ for an additional $120 \mathrm{~h}$. Cell viability was determined by the MTS method (Promega).

To study AhR pathway inhibition, cells were pre-incubated for $1 \mathrm{~h}$ with AhR specific antagonists, either $\alpha$-naphthoflavone $(\alpha-\mathrm{NF}, 1 \mu \mathrm{M})$ or 4,7-phenantroline(PHT, $10 \mu \mathrm{M})$ (Sigma-Aldrich).

AhR western blot analysis. Cells were seeded at $6 \times 10^{5}$ in a T25 flask $24 \mathrm{~h}$ before treatment with $1 \mu \mathrm{M} \mathrm{AF}$ for $30 \mathrm{~min}, 3$ and $6 \mathrm{~h} ; 0.1 \%$ DMSO for $6 \mathrm{~h}$ or $10 \mathrm{nM}$ TCDD for $1 \mathrm{~h}$. Following treatment, the cells were harvested, lysed and centrifuged using a commercial kit (NER-PER, Pierce Biotechnology) in order to isolate cytosolic and nuclear fractions. Protein concentration was determined using the Bradford assay.

Both fractions were analyzed by western blot analysis. Proteins $(30 \mu \mathrm{g})$ were resolved on $8 \%$ SDS-polycacrylamide gel and transferred electroforetically onto nitrocellulose membranes for $120-140 \mathrm{~min}$ at $0.3 \mathrm{~A}$. The membranes were blocked with blocking buffer consisting of 5\% non-fat dry milk in $1 \%$ Tween-20 in $20 \mathrm{mM}$ TBS (TTBS) (pH 7.5) for $1 \mathrm{~h}$ at room temperature, then cells were incubated overnight at $4^{\circ} \mathrm{C}$ with rabbit primary antibody against human AhR (sc-5579; Santa Cruz Biotechnology) at a dilution of 1:1000 in TTBS. The membranes were then incubated with goat anti-rabbit IgG-HRP secondary antibody (sc- 2004) at a dilution of 1:5000 in TTBS for $1 \mathrm{~h}$ at room temperature and visualized using the enhanced chemiluminescence detection system. Autoradiographies were scanned and quantified by densitometry using GelPro Analyzer 4 software. Equal protein loading in both fractions was confirmed by reproving the membranes with a mouse antiactin antibody (A5441; Sigma).

Transient transfections. Cells were plated in 24-well plates at a concentration of $1 \times 10^{5}$ cells per well. After $24 \mathrm{~h}$ the cells were transfected using Lipofectamine 2000 (Invitrogen) with $0.5 \mu \mathrm{g}$ Renilla reniformis luciferase and $1.5 \mu \mathrm{g}$ pTX.Dir plasmid [two XRE sequences extending from nucleotides -1026 to -999 relative to the transcription start site of the rat CYP1A1 inserted into a vector containing the herpex simplex virus timidine kinase (TK) promoter and the luciferase reporter gene] (11) or pT81 (same reporter plasmid without the XRE sequence, used as the negative control) (12). After $24 \mathrm{~h}$, the transfected cells were treated with $10 \mathrm{nM}$ TCDD; $10 \mu \mathrm{M}$ AF or $10 \mu \mathrm{M}$ AF plus $1 \mu \mathrm{M} \alpha-\mathrm{NF}$, as shown in the figures. The control cells were transfected with pTX.Dir and treated with DMSO $(0.1 \%)$. 
After $18 \mathrm{~h}$ of treatment, luciferase activity was measured by the dual luciferase assay system (Promega) following the manufacturer's instructions. Transfection efficiency was monitored by $R$. reniformis luciferase activity using the pRL-TK plasmid as an internal control.

Induction of apoptosis by AF. Cells were seeded at $2.5 \times 10^{5}$ on a $35-\mathrm{mm}$ dish overnight and then exposed to DMSO $(0.1 \%)$ or $1 \mu \mathrm{M}$ AF for $24 \mathrm{~h}$. Floating cells contained in the supernatant were collected by cytocentrifugation. Once fixed, cells were stained with 0.4\% 4,6-diamidino-2-phenylindole (DAPI) and observed under a fluorescence microscope. The experiments were repeated at least three times. Condensed and fragmented nuclei were considered apoptotic.

Apoptotic/necrotic cells were also determined by acridine orange/ethidium bromide staining. Tumor cells were seeded on cover slides in 6-well plates, treated with $1 \mu \mathrm{M} \mathrm{AF}$ for $24 \mathrm{~h}$, and then washed. The AhR inhibitor, $\alpha-\mathrm{NF}(1 \mu \mathrm{M})$, was also added. After $24 \mathrm{~h}$, cells adhered to the cover slides and present in the supernatants were stained with a mix containing acridine orange $(10 \mu \mathrm{g} / \mathrm{ml})$ plus ethidium bromide $(10 \mu \mathrm{g} / \mathrm{ml})$. Cells with green fluorescence and condensed chromatin were recorded as apoptotic, while orange cells with lax chromatin were recorded as necrotic.

Determination of phosphorylated P53 (p-P53) and p21. Western blot analysis was performed as previously described $(13,14)$ using antibodies against p-P53 (Ser392, AB4060; Chemicon) at a dilution of 1:600 and P21 (Cell Signaling Technology; Cat no. 2946) at a dilution of 1:500. p-P53 and P21 western blot analyses were performed using total lysates of TK-10 cells treated with AF $(1 \mu \mathrm{M})$ for $30 \mathrm{~min}, 1,3,6,24$ and $48 \mathrm{~h}$.

Determination of caspase 3 and poly(ADP-ribose) polymerase $(P A R P)$. Western blot analysis was performed using total lysates of TK-10 cells treated with AF $(1 \mu \mathrm{M})$ for $30 \mathrm{~min}, 1 \mathrm{~h}$, $3 \mathrm{~h}, 6 \mathrm{~h}, 24 \mathrm{~h}$ and $48 \mathrm{~h}$ using caspase 3 antibody of Santa Cruz (sc-7841) dilution 1:200 and PARP BD Pharmingen (556494) dilution 1:500.

Statistical analysis. Statistical significance between three or more groups was calculated by one-way analysis of variance (ANOVA) followed by Tukey's test. To compare two groups, the unpaired Student's t-test with Welch correction was used. Statistical analysis was performed using GraphPad InStat version 3.06 for Windows 95 (GraphPad Software Inc., San Diego, CA, USA; www.graphpad.com). Designations for statistical significance were ${ }^{*} \mathrm{p}<0.05$ and ${ }^{* *} \mathrm{p}<0.01$.

\section{Results}

AF-induced citotoxicity in human renal cancer cell lines is mediated by $A h R$. In this study, we measured the sensitivity of cells to AF as an in vitro regression (shown by a decrease in cell metabolism measured by the MTS assay).

The incubation of TK-10, SN12-C, Caki-1 and A498 cells with $1 \mu \mathrm{M}$ AF for five days induced a decrease in cell viability

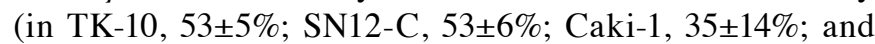
A-498 cells, $38 \pm 16 \%$, with respect to the control which was considered 100\%). The effect was dependent on drug concentration. In contrast, $\mathrm{ACHN}$ cells were not sensitive to AF as growth inhibition was not observed at any drug concentration (a decrease of $4 \pm 4 \%$ of control) (Fig. 2A).

In order to investigate whether sensitivity to AF of renal cancer cells is mediated by AhR activation, we pre-incubated the cells with the AhR specific inhibitor, $\alpha-\mathrm{NF}(1 \mu \mathrm{M})$, for $1 \mathrm{~h}$ prior to treatment with $1 \mu \mathrm{M}$ AF plus $\alpha-N F$. The AhR inhibitor partially reduced the citotoxic effects of the drug (a decrease

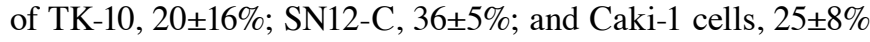
with respect to the control). The sensitivity of A498 cells was not diminished by pre-incubation with the AhR inhibitor: (38 $\pm 16 \%)$ (Fig. 2B).

Additional experiments indicated that pre-incubation of TK-10 cells with other AhR inhibitor (PHT, $10 \mu \mathrm{M})$ partially prevented the AF citotoxic effect. This effect was observed by phase contrast microscopy (Fig. 2C) and by MTS assay (data not shown).

AF induces AhR translocation in drug-sensitive renal cancer cells. The results shown in Fig. 2 are consistent with the idea that AF may be an agonist ligand for AhR, inducing both the translocation of AhR to the nucleus with subsequent binding to XRE sequences and the transcriptional activation of target genes, including CYP1A1 and CYP1B1. To assess this possibility, using TK-10, Caki-1 and SN12-C renal cancer cell lines, which are sensitive to AF, we examined whether this drug can activate the AhR signaling pathway, resulting in AhR translocation from the cytoplasm to the nucleus. The effect of AF $(1 \mu \mathrm{M})$ on the subcellular distribution of the immunoreactive $\mathrm{AhR}$ protein was studied by western blot analysis. As demonstrated in Fig. 3, in TK-10, Caki-1 and SN12-C cells treated with DMSO only, the cytoplasmic fraction contained relatively high levels of the AhR protein compared with the nuclear fraction. In contrast, after treatment with $1 \mu \mathrm{M}$ AF for 0.5 to $6 \mathrm{~h}$ or TCDD $(10 \mathrm{nM})$ for $1 \mathrm{~h}$ (as the positive control), the immunoreactive AhR protein translocated to the nuclear fraction. The results were confirmed by immunofluorescence studies (data not shown).

We then wished to investigate whether AF can cause AhR nuclear translocation in an insensitive cell line, such as ACHN. The effect of AF $(1 \mu \mathrm{M})$ on the subcellular distribution of immunoreactive AhR protein was assessed by western blot analysis. As demonstrated in Fig. 3, in ACHN cells treated with DMSO only, cytoplasmic and nuclear fractions contained high levels of the AhR protein. After treatment with $1 \mu \mathrm{M} \mathrm{AF}$, for 0.5 to $6 \mathrm{~h}$, immunoreactive AhR did not translocate to the nuclear fractions. However, TCDD treatment $(10 \mathrm{nM})$ for $1 \mathrm{~h}$ caused the nuclear translocation of AhR.

AF induces activation of CYPIA1-related promoter sequences. The CYP1A1 and CYP1B1 promoters are regulated by AhR, which forms a heterodimer with Arnt. Binding of the complete dimmer to XREs in the promoter region mediates the transcription of AhR-responsive genes, including CYP1A1 and CYP1B1. Thus, we sought to define whether AF activates AhR, causing CYP1A1 transcription. TK-10, SN12-C, Caki-1 and ACHN cells were transfected with an XRE luciferase reporter construct (pTX.Dir), and as the control, the same reporter construct without XRE elements (pT81) was used. Cells were then treated with $0.1 \%$ DMSO, $0.1-10 \mu \mathrm{M}$ AF or $10 \mathrm{nM}$ TCDD. 
A

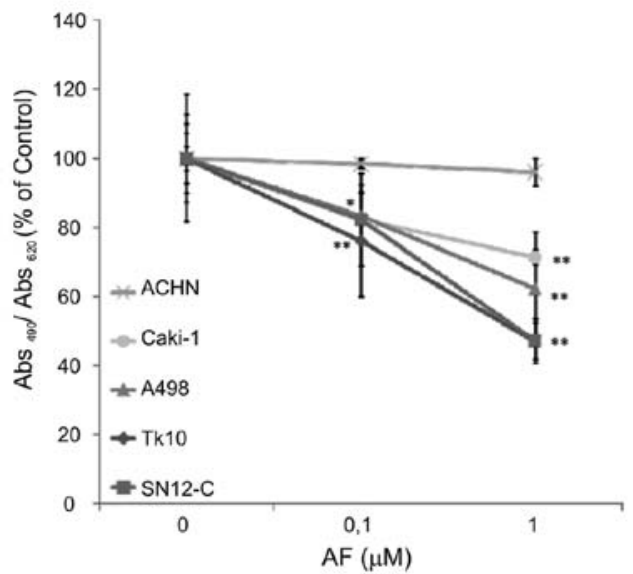

B

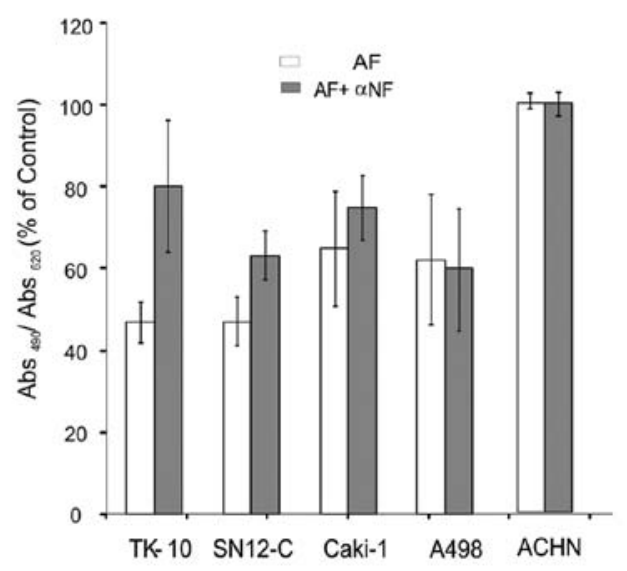

C
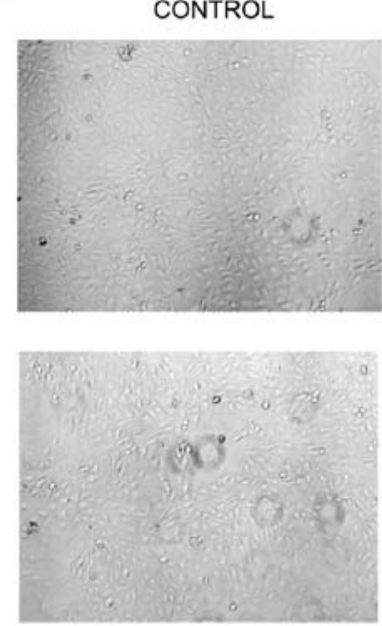

PHT
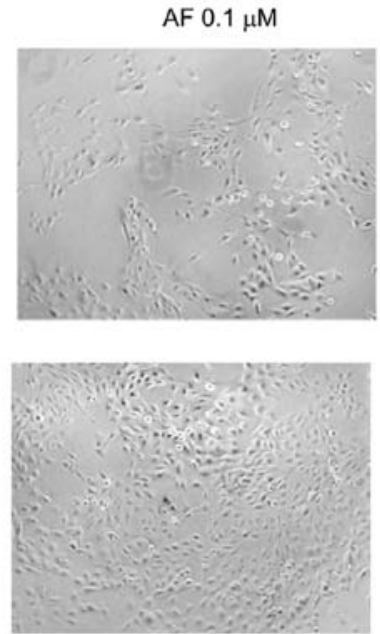

$\mathrm{PHT}+\mathrm{AF} 0.1 \mu \mathrm{M}$
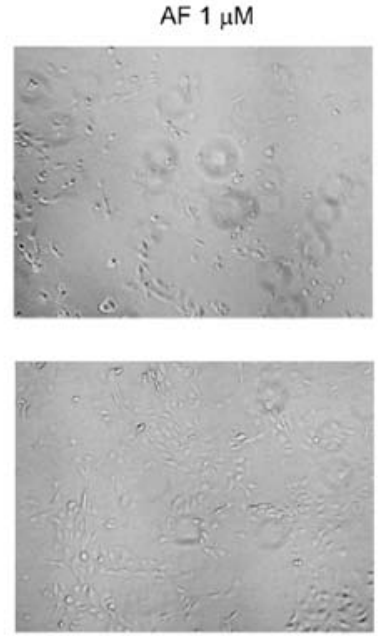

$\mathrm{PHT}+\mathrm{AF} 1 \mu \mathrm{M}$

Figure 2. Sensitivity to aminoflavone (AF) depends on aryl hydrocarbon receptor (AhR) activity. (A) Inhibition of viability of human renal cancer cell lines after treatment with AF. Cells were incubated with AF or DMSO $(0.1 \%)$ for five days. Cellular viability was then evaluated by MTS assay. The values represent the average of three independent experiments (TK-10 and ACHN, n=12; A498, Caki-1 and SN12-C, $\mathrm{n}=10$ ). ${ }^{*} \mathrm{p}<0.05$; ${ }^{* *} \mathrm{p}<0.01$, compared with untreated cells. The results were analyzed by ANOVA and Tukey test. (B) Sensitivity to AF was partially prevented by the AhR inhibitor, $\alpha$-naphthoflavone ( $\alpha$-NF). Cells were incubated with AF $(1 \mu \mathrm{M})$ for five days alone or pre-treated for $1 \mathrm{~h}$ with $\alpha$-NF $(1 \mu \mathrm{M})$ and then treated with AF (1 $\mu \mathrm{M})$ plus $\alpha$-NF $(1 \mu \mathrm{M})$ for five days. Cellular viability was then evaluated by MTS assay. The values represent the average of three independent experiments $(\mathrm{n}=12){ }^{* *} \mathrm{p}<0.01 \mathrm{compared}$ with cells incubated without the AhR inhibitor. (C) Sensitivity to AF was partially prevented by the AhR inhibitor, PHT. TK-10 cells were incubated with PHT (10 $\mu$ M) alone for five days or pre-treated with PHT $(10 \mu \mathrm{M})$ for $1 \mathrm{~h}$ and then treated with AF + PHT for five days. Cells were photographed using a phase contrast microscope. Representative fields of three independent experiments are shown.

As shown in Fig. 4A, in TK-10 cells transfected with pTX.Dir, a 1.8-fold induction of luciferase activity was observed when cells were treated with $10 \mu \mathrm{M}$ AF. In the other AF-sensitive cell line, SN12-C, transfected with pTX.Dir, AF $(10 \mu \mathrm{M})$ caused a 3.5-fold increase in luciferase activity compared to the control. Also Caki-1 cells, sensitive to the drug, showed a 2.6-fold induction of luciferase activity. ACHN cells, insensitive to the drug, did not show an induction in luciferase activity when transfected with pTX.Dir and treated with AF $(10 \mu \mathrm{M})$. Cells transfected with the empty vector, pT81, and treated with $10 \mu \mathrm{M}$ AF did not shown an induction in luciferase activity. These findings clearly demonstrate that AF induces the activation of promoter sequences known to respond to AhR-mediated signals in sensitive cell lines. This is in accordance with the interaction of protein complexes induced by treatment with $\mathrm{AF}$ through the XRE sequence of the CYP1A1 promoter.

In order to confirm that the increase in luciferase activity observed in TK-10 cells after treatment with AF was due to the specific interaction of AhR protein complexes with the XRE sequence of the CYP1A1 promoter, cells were transfected with an XRE luciferase reporter construct (pTX.Dir) and then treated with $0.1 \%$ DMSO, $10 \mu \mathrm{M}$ AF or $10 \mu \mathrm{M}$ AF plus $1 \mu \mathrm{M}$ $\alpha-N F$. As shown in Fig. 4B, TK-10 cells transfected with pTX. Dir, and treated with $10 \mu \mathrm{M}$ AF, exhibited a 1.8-fold induction of luciferase activity. In cells pre-treated for $1 \mathrm{~h}$ with the AhR inhibitor, $\alpha-\mathrm{NF}(1 \mu \mathrm{M})$, and then treated for $18 \mathrm{~h}$ with AF plus 
TK-10

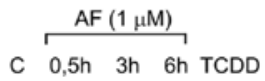

Cytosolic AhR

NuclearAhR
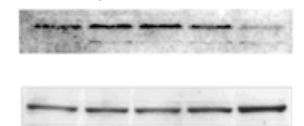

$122 \mathrm{kDa}$
Caki-1

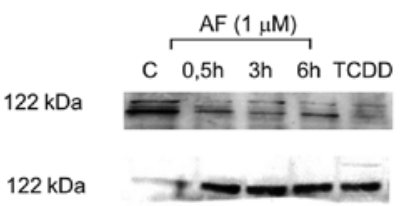

ACHN

$\mathrm{AF}(1 \mu \mathrm{M})$

C $0,5 \mathrm{~h} \quad 3 \mathrm{~h} \quad 6 \mathrm{~h}$ TCDD

$122 \mathrm{kDa}$

$122 \mathrm{kDa}$
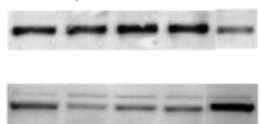

Nuclear AhR
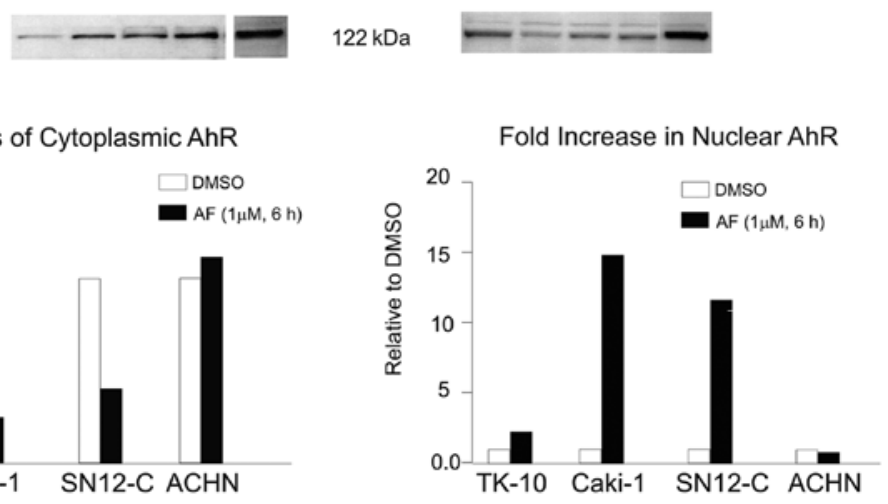

Figure 3. Effect of aminoflavone (AF) on aryl hydrocarbon receptor (AhR) nuclear translocation in renal cancer cell lines. TK-10, Caki-1, SN12-C and ACHN cells were incubated with $\mathrm{AF}(1 \mu \mathrm{M})$ for the indicated times, DMSO $(0.1 \%)$ for $6 \mathrm{~h}$ or TCDD $(10 \mathrm{nM})$ for $1 \mathrm{~h}$ as a positive control. Nuclear and cytosolic fractions were isolated using a commercial kit and analyzed for AhR content by western blot analysis. Representative western blots are shown in the top panel. All western blot analyses were performed three times for each cell line showing the same pattern of AhR subcellular distribution.
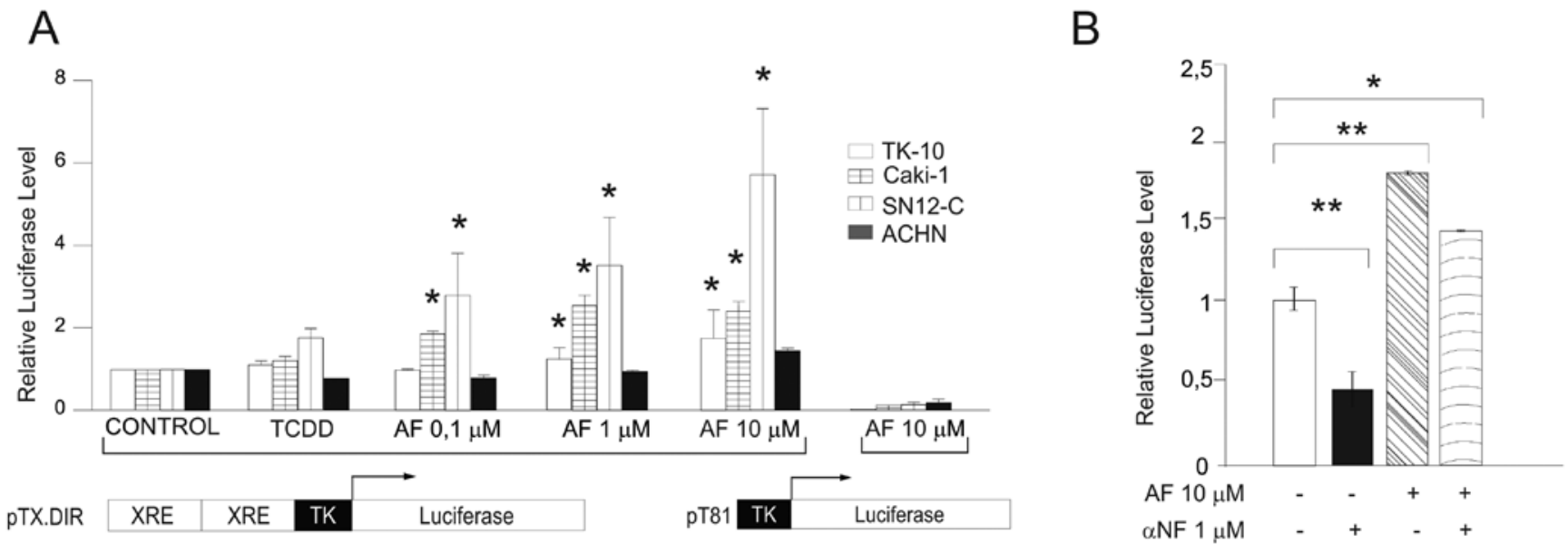

Figure 4. Aminoflavone (AF) induces the activation of CYP1A1-related promoter sequences in sensitive renal cancer cells. (A) Cells were transfected with Lipofectamine 2000 (Invitrogen) and a plasmid containing XRE [aryl hydrocarbon receptor (AhR) consensus sequences] upstream of the luciferase reporter gene and a second plasmid containing R. reniformis luciferase gene as the internal control. After $24 \mathrm{~h}$, cells were incubated with AF for $18 \mathrm{~h}$. Luciferase activity was determined using the dual luciferase reporter assay system (Promega). The values represent the average of three independent experiments ( $\mathrm{n}=4$ ), ${ }^{*} \mathrm{p}<0.05$ compared with cells incubated without AF. (B) AF-induced activation of CYP1A1-related promoter sequences in TK-10 was inhibited by pre-treatment with $\alpha$-NF. TK-10 cells were transfected with Lipofectamine 2000 and a plasmid containing XRE (AhR consensus sequences) upstream of the luciferase reporter gene and a second plasmid containing R. reniformis luciferase gene as the internal control. After $24 \mathrm{~h}$, cells were incubated with $\mathrm{AF}$ for $18 \mathrm{~h}$, or, pre-treated $1 \mathrm{~h}$ with $1 \mu \mathrm{M} \alpha-\mathrm{NF}$ and then treated $18 \mathrm{~h}$ with AF plus $1 \mu \mathrm{M} \alpha-\mathrm{NF}$. Luciferase activity was determined using the dual luciferase reporter assay system. The values represent the average of three independent experiments $(n=4),{ }^{*} \mathrm{p}<0.05$ or ${ }^{* *} \mathrm{p}<0.01$ compared with cells incubated without AF.

$\alpha$-NF, we observed a 1.3-fold induction of luciferase activity. These results confirm that AhR is involved in the increase in XRE luciferase activity.
AF-induced apoptosis is mediated by AhR. We previously reported that AF induces apoptosis in AF-sensitive human renal tumor cell lines. Treatment of cell lines most sensitive to 
A
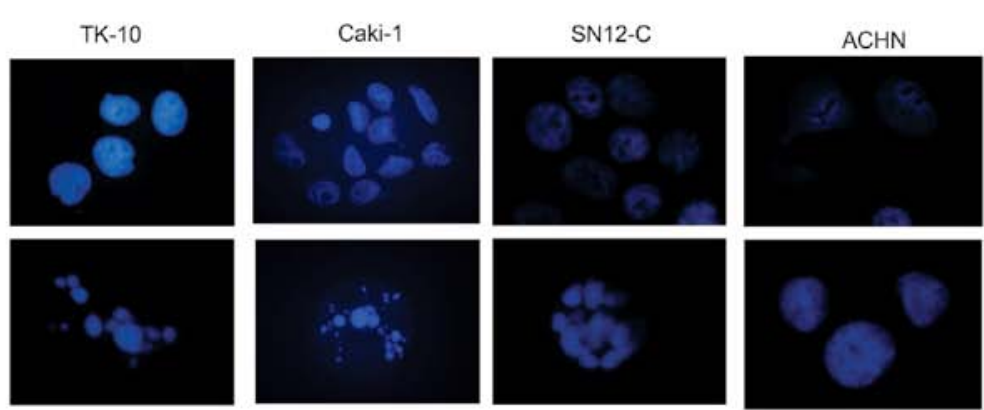

B
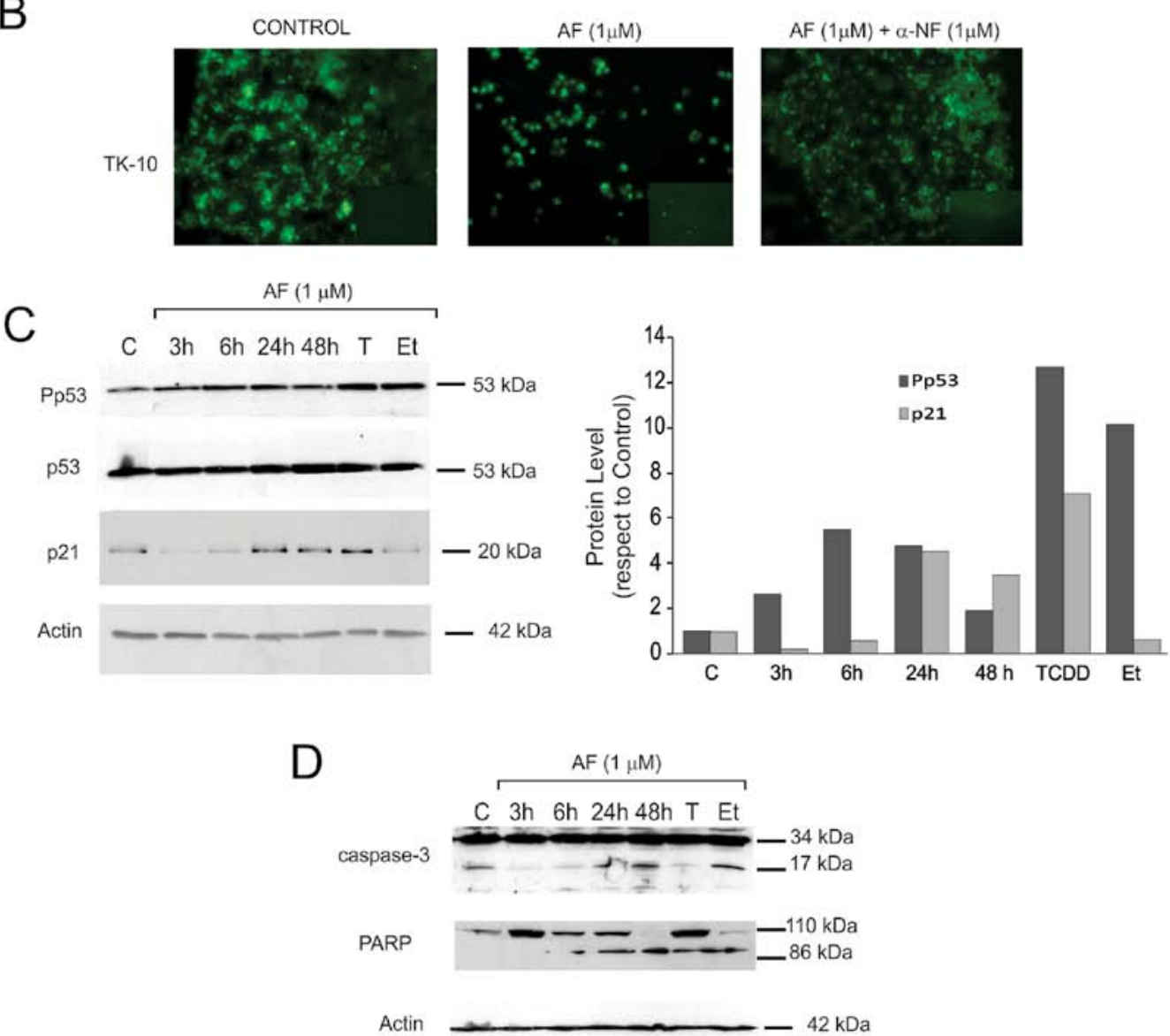

Figure 5. Aminoflavone (AF) induces apoptosis mediated by aryl hydrocarbon receptor (AhR) in sensitive renal tumor cells. (A) Cells were incubated with $1 \mu \mathrm{M}$ AF or DMSO $(0.1 \%)$ for $72 \mathrm{~h}$. Then, non-adherent cells were obtained by cytocentrifugation in the culture medium. Once fixed, cells were stained with DAPI and observed under a fluorescence microscope. Condensed and fragmented nuclei were considered apoptotic. Representative fields are shown. (B) Acridine orange/ ethidium bromide staining of apoptotic cells in TK-10 monolayer. Cells were treated with DMSO (control), AF (1 $\mu \mathrm{M})$ for $24 \mathrm{~h}$ or pre-incubated for $1 \mathrm{~h}$ with $\alpha$-NF followed by $24 \mathrm{~h}$ of AF+ $\alpha$-NF $1 \mu \mathrm{M}$ (upper panel). (C) Effect of AF on p53 signaling pathway in TK-10 cells. Cells were incubated with $1 \mu \mathrm{M}$ AF during indicated times or TCDD (T) $10 \mathrm{nM}$ for $1 \mathrm{~h}$. Cells treated with $10 \mu \mathrm{M}$ Etoposide (Et) were used as a positive control for apoptosis. Whole cell extracts were obtained and subjected to SDS-PAGE and western blotting with p-P53 and P21 antibodies. p-P53, phosphorylated form of P53. (D) Effect of AF on caspase 3 activation and PARP cleavage in TK-10 cells. Cells were incubated with $1 \mu \mathrm{M}$ AF for the indicated times. Whole cell extracts were obtained and subjected to SDS-PAGE and western blotting with specific antibodies was performed.

the drug resulted in the greatest elevation in caspase-mediated cleavage of cytokeratin $18(6)$.

In this study, we further investigated the mechanisms leading these cells to apoptosis. We observed the formation of apoptotic bodies in TK-10, Caki-1 and SN12-C cell lines (Fig. 5A); in the AF-resistant cell line, ACHN, we did not observe apoptotic body formation.

We then sought to determine whether AF-induced apoptosis is dependent on AhR. TK-10 cells were seeded and $24 \mathrm{~h}$ later a treatment with $1 \mu \mathrm{M}$ AF was performed. In this experiment, apoptotic but not necrotic cells were observed (Fig. 5B). The percentage of apoptotic cells (with condensed chromatin) that remained adhered after AF treatment was higher than in controls. This result was diminished by pre-incubation with the AhR inhibitor, $\alpha-N F$. Apoptotic cells were also recorded in the supernatants showing similar results. These data suggest that AhR is involved in the AF pro-apoptotic activity. 
In order to investigate whether AF treatment causes P53 phosphorylation in TK-10 cells as a result of its pro-apoptotic action, we carried out a western blot analysis with these cells treated with $1 \mu \mathrm{M}$ AF for 3-48 h. We observed a 2.6-fold increase in p-P53 levels at $3 \mathrm{~h}$ of treatment. At $6 \mathrm{~h}$ of treatment we observed a 5.5 -fold increase and at $24 \mathrm{~h}$ of treatment a 4.7-fold increase in p-P53 levels; after that time p-P53 levels slowly decreased. Considering that P53 can induce P21 activation and cell cycle arrest, we studied P21 protein levels. We found a 4.5-fold increase in P21 protein levels at $24 \mathrm{~h}$ and a 3.5-fold increase at $48 \mathrm{~h}$ of treatment (Fig. 5C). This result suggests that the drug may also cause cell cycle arrest in renal cancer cells.

In order to investigate whether AF-induced apoptosis involves caspase 3 activation, we analyzed caspase 3 protein levels after treatment with the drug. Between 24 and $48 \mathrm{~h}$ of treatment we found caspase 3 cleavage from its native form of $32 \mathrm{kDa}$ into 17 and $12 \mathrm{kDa}$ subunits, indicating the activation of this enzyme by the drug (Fig. 5D). Finally, considering that during apoptosis, PARP is cleaved by caspase 3 , we studied PARP cleavage after treatment with AF. We observed the cleavage of PARP from the native 116 to $85 \mathrm{kDa}$ between 6 to $48 \mathrm{~h}$ of treatment (Fig. 5D).

$A F$ induces activation of $A h R$ in a sensitive papillary renal carcinoma cell strain. In another experiment, we used a cell strain obtained from a renal papillary tumor of a patient, termed as 112. We treated the cells with AF for five days. We then measured citotoxicity using an MTS assay. AF (500 nM) caused a decrease in cell viability of $90 \%$ of the control (considered 100\%). This decrease in cell viability diminished to $30 \%$ when cells were pre-treated with $\alpha-\mathrm{NF}$ followed by incubation with $\mathrm{AF} / \alpha-\mathrm{NF}$. These results indicate that AhR mediates the effect of AF in this papillary tumor sensitive to the drug (Fig. 6A and B).

We then investigated whether AF causes AhR nuclear translocation in 112 cells. As demonstrated in Fig. 6C, in the cells treated with DMSO only, we found high levels of the AhR protein in cytosolic and nuclear fractions. After treatment with $1 \mu \mathrm{M}$ AF or $10 \mathrm{nM}$ TCDD, we found immunoreactive AhR protein increased in the nuclear fraction and decreased in the cytosol (Fig. 6C).

We then wished to determine whether AF-induced apoptosis is dependent on AhR in this papillary cell strain. Cells were seeded and $24 \mathrm{~h}$ later a treatment with $1 \mu \mathrm{M}$ AF was performed. In this experiment, apoptotic but not necrotic cells were observed. The percentage of cells with chromatin condensation (apoptotic) that remained adhered after AF treatment was higher compared to the control. This result was abrogated by pre-incubation with the AhR inhibitor $\alpha-\mathrm{NF}$ suggesting that AhR is involved in the AF pro-apoptotic activity (Fig. 6D). Also, DAPI nuclear staining showed the formation of apoptotic bodies after treatment of the 112 cell strain with AF (Fig. 6E).

\section{Discussion}

The main goal of this study was to understand more deeply the mechanism by which AF causes toxicity in human renal tumor cells. Our results show that the antiproliferative effect of $\mathrm{AF}$ is mediated by the activation of $\mathrm{AhR}$ in human renal cancer sensitive cell lines, which present CYP1A1 induction after treatment with AF.

In a previous study, we showed that certain cell lines, such as Caki-1, TK-10 and A498, are very sensitive to AF (6). They showed high levels of apoptosis, measured by cytokeratin 18 cleavage after treatment with AF. In the present study, we found that treatment with AF resulted in AhR signaling pathway activation in Caki-1 and TK-10 cells (Figs. 2-5). These results are in agreement with our previous observations showing high induction of CYP1A1 after treatment with $0.1 \mu \mathrm{M}-1 \mu \mathrm{M}$ of AF in these two cell lines (6). Our results confirm that the activation of AhR leads to CYP1A1 induction, which induces AF metabolism to reactive intermediates that cause DNA damage and apoptosis.

In this study, we showed that AF pro-apoptotic action in TK-10 cells is mediated by AhR. However, we found that incubation with the AhR inhibitor, $\alpha-N F$, prior to treatment with AF, decreased the number of apoptotic bodies formed (Fig. 5). This indicates that the AF-induced apoptosis is mediated by AhR activation. Furthermore, we studied the molecular pathway involved in AF-induced apoptosis, and we observed the induction of p-P53 and P21 levels after treatment with $1 \mu \mathrm{M}$ AF in these cells between 3 and $6 \mathrm{~h}$, which confirms that this compound causes apoptosis and presumably cell cycle arrest (Fig. 5). This finding is consistent with the induction of p-P53 and P21 that was previously reported after AF treatment of MCF-7 breast cancer cells (14). As a tumor suppressor, the best understood function for P53 is to mediate cell cycle arrest or apoptosis after DNA damage, thereby preventing the propagation of damaged cells (15). Future flow cytometry studies will confirm whether AF causes apoptosis and cell cycle arrest in AF renal sensitive cells in an AhR-dependent manner.

In addition, we found caspase 3 activation and consequent PARP cleavage after treatment with AF in TK-10 cells (Fig. 5) which is consistent with the DNA damage and apoptosis caused by this antitumor agent. Considering that PARP is an enzyme implicated in DNA damage and repair mechanisms, and that PARP is inactivated by caspase cleavage, leading cells to apoptosis, our results confirm that AF induces apoptosis in TK-10 cells. It has been shown that AF induces $\gamma \mathrm{H} 2 \mathrm{AX}$ phosphorylation consistent with the induction of DNA single-strand breaks and DNA-protein cross-links in MCF-7 cells after treatment with AF (13). Furthermore, caspase 3-dependent apoptotic body formation was observed in MCF-7 breast cancer cells after treatment (8), which is consistent with our observation in renal cancer cells.

The A498 cell line showed remarkable toxicity after treatment with AF. This effect did not change when we preincubated the cells with the AhR inhibitor, $\alpha-\mathrm{NF}$, indicating that this receptor does not mediate the AF toxic effects in these cells (Fig. 2). This was in accordance with our previous observations indicating that the drug induced high levels of apoptosis in this cell line, but it did not cause CYP1A1 induction (6). It is likely that other signaling pathways independent of AhR are involved in the toxicity observed in these cells. For instance, the modulation of survival pathways, such as Akt/PI3K has been reported following AF treatment (14).

In our previous study, SN12-C cells showed low levels of CYP1A1 induction after treatment with AF; however, the levels of apoptosis were relatively high (6). In this study, these cells 


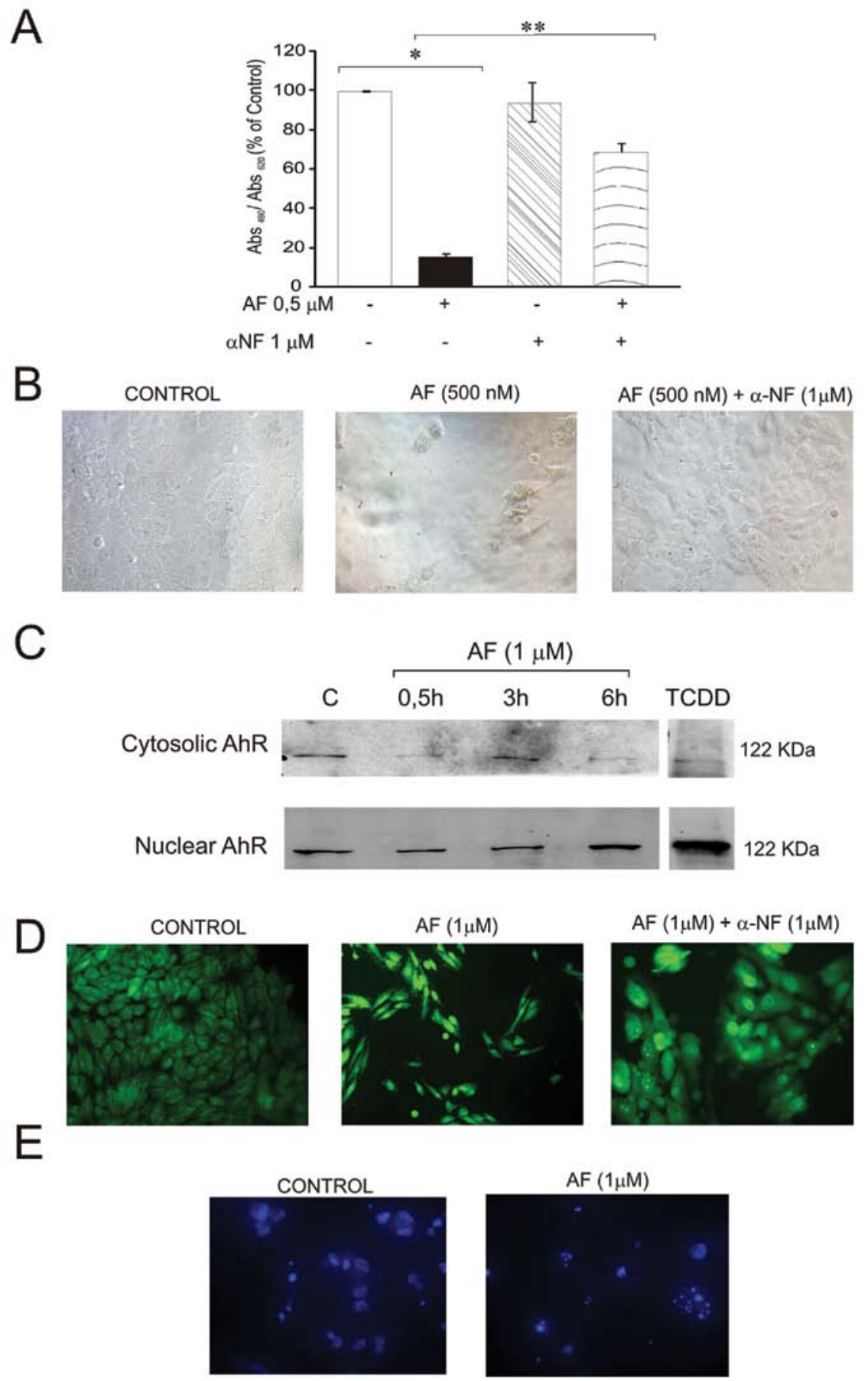

Figure 6. Aminoflavone (AF) effect on the 112 papillary cell strain. Role of the aryl hydrocarbon receptor (AhR) pathway. Cells were incubated with $1 \mu \mathrm{M}$ $\mathrm{AF}$ and/or $\alpha-\mathrm{NF}(1 \mathrm{mM}$ ) for 5 days. (A) Inhibition of viability of 112 human renal cell strain after treatment with AF. Cells were incubated with AF or DMSO $(0.1 \%)$ for 5 days. Then, cellular viability was evaluated by MTS assay. The values represent the average of three independent experiments $(\mathrm{n}=10)$. ${ }^{*} \mathrm{p}<0.05$ comparing with untreated cells; ${ }^{* *} \mathrm{p}<0.01$ compared with cells without AhR inhibitor. Results were analyzed by ANOVA and the Tukey test. (B) Then, cells were photographed using a phase contrast microscope. Representative fields of one experiment are shown. (C) Cells were incubated with $\mathrm{AF}(1 \mu \mathrm{M})$ for the indicated times, DMSO $(0.1 \%)$ for $6 \mathrm{~h}$ or TCDD $(10 \mathrm{nM})$ for $1 \mathrm{~h}$ as the positive control. Nuclear and cytosolic fractions were isolated using a commercial kit and analyzed for AhR content by western blotting. Representative western blots are shown. (D) Acridine orange/ethidium bromide staining of apoptotic cells in 112 monolayer. Cells were treated with DMSO (control), $\mathrm{AF}(1 \mu \mathrm{M})$ for $24 \mathrm{~h}$ or pre-incubated for $1 \mathrm{~h}$ with $\alpha-\mathrm{NF}$ followed by $24 \mathrm{~h}$ of $1 \mu \mathrm{M} \mathrm{AF}+\alpha-\mathrm{NF}$. (E) Cells were incubated with $1 \mu \mathrm{M}$ AF or DMSO $(0.1 \%)$ for $72 \mathrm{~h}$. Then, non-adherent cells were obtained by cytocentrifugation in the culture media. Once fixed, cells were stained with DAPI and observed under a fluorescence microscope. Condensed and fragmented nuclei were considered apoptotic. Representative fields of one experiment are shown.

showed high sensitivity measured by MTS assays, which was partially abrogated by pre-incubation with $\alpha-\mathrm{NF}$ (Fig. 2B). Furthermore, we showed AhR pathway activation in these cells. Treatment with AF caused the translocation of AhR to the nucleus (Fig. 3) and the induction of AhR transcriptional activity (Fig. 4).

In addition, experiments were performed with the renal cell strain 112, which was derived from a papillary tumor sensitive 
to AF. These cells also showed AhR activation (Fig. 6) by AF, which was in agreement with CYP1A1 induction previously observed in this renal cell strain (6). This experiment was performed with AF NSC686288 and AFP464 NSC710464 and we obtained the same results using both compounds; however, we found a more clear effect using AF NSC710464 dissolved in 5\% dextrose water (which was more soluble in the cell culture medium). The AF toxic effect on these cells was almost abolished by pre-incubation with $\alpha-N F$, showing that AhR mediated this effect. This agent caused apoptotic body formation observed by acridine orange/ethidium bromide and DAPI nuclear staining. Additionally we observed AhR nuclear translocation after treatment with the drug. In our previous report, we indicated that papillary renal tumors are more sensitive to AF than clear cell tumors (6). The enhanced activity of AF against the papillary variant of renal cell carcinoma is of special value. Except for temsirolimus and sunitinib, (both have proven their efficacy in the treatment of non-clear cell kidney cancer and are recomended for clinical use), there are litlle or no data regarding the safety and efficacy of the new target drugs in papillary histology and there is a need for the development of new effective therapies (16). However, AhR activation by AF has to be confirmed in future studies using other papillary and clear cell carcinoma tumors.

In contrast to the other cell lines, $\mathrm{ACHN}$ cells showed resistance to AF treatment which was associated with the lack of induction of CYP1A1 and CYP1B1 transcription (6). In the present report, we demonstrate that AhR activation does not occur in these cells in response to AF (Figs. 4 and 5). We hypothesize that this may lead to the lack of activation of CYP1A1 with the consequent lack of ability of these cells to metabolize AF. In ACHN cells treated with DMSO only, AhR was present in the cytosol and nucleus. After treatment with $1 \mu \mathrm{M}$ AF, for 0.5 to $6 \mathrm{~h}$, immunoreactive AhR protein levels do not change in the cytosolic and nuclear fractions. Of note, we observed high levels of $\mathrm{AhR}$ in the nucleus even in the control, indicating that AhR may be constitutively nuclear in this cell line. This is a common pattern of AhR subcellular distribution in cell lines of different origin resistant to AF (5). A suitable hypothesis may be that nuclear export or degradation signals may be altered leading to inappropriate AhR recycling. However, this has to be tested in future studies. A different AhR variant present in ACHN cells could explain the fact that AF does not induce translocation of the receptor in this cell line. Also, differential expression of proteins that regulate AhR activation, such as HSP90, may induce resistance to AF. Additionally, differential activation of the survival signaling pathways, such as different isoforms of Akt, or differential expression of NF-kB may cause resistance to AF; however this hypothesis remains to be investigated.

In this study, we used a panel of renal cancer cell lines with different von Hippel Lindau (VHL) status. Our results indicate that sensitivity or resistance to AF is independent of VHL status and HIF expression. This is significant as it widens the variety of renal tumors that can be effectively treated with this agent. Recently, it has been demonstrated that AF inhibits HIF1 $\alpha$ expression in an AhR-independent fashion in certain breast, renal and ovarian cancer cell lines (17).

Furthermore, AF may cause not only apoptosis but also different types of cell death in renal cancer cells. Recent studies have demonstrated that certain drugs induce autophagy rather than apoptosis in renal cancer cells with mutated VHL and that this toxicity is independent of HIF1 and HIF2 status (18). If this is the case with AF, it has to be clarified in future studies.

In this study, we demonstrate for the first time that the AhR signaling pathway activation mediates AF antitumor action in renal cancer. The activation of this signaling pathway may be a new molecular target for the treatment of these tumors, different from others currently used in the clinic. Finally, AhR translocation and activation may be used as a biomarker in tumor biopsies to predict sensitivity to treatment with AF of renal tumors of different histological types. This could be incorporated into phase II clinical trials, together with other markers of sensitivity, such as the induction of CYP1A1, high covalent binding of metabolites and apoptosis, for the selection of patients that potentially could respond to the treatment with this agent $(5,6)$.

\section{Acknowledgements}

This study was carried out with the financial support of Tigris Pharmaceuticals Inc., USA and a grant from Fundacion Florencio Fiorini para Investigación en Ciencias Biomédicas, Argentina.

\section{References}

1. Jemal A, Siegel R, Ward E, Murray T, Xu J, Smigal C and Thun M: Cancer Statistics, 2006. CA Cancer J Clin 56: 106-130, 2006.

2. Escudier B, Eisen T, Stadler WM, et al: Sorafenib in advanced clear-cell renal-cell carcinoma. N Engl J Med 356: 125-134, 2007.

3. Escudier B, Pluzanska A, Koralewski P, et al: Bevacizumab plus interferon alfa-2a for treatment of metastatic renal cell carcinoma: a randomised, double-blind phase III trial. The Lancet 370: 2103-2111, 2007.

4. Motzer R, Hutson TE, Tomczak P, et al: Sunitinib versus interferon alfa in metastatic renal-cell carcinoma. N Engl J Med 356: 115-124, 2007.

5. Loaiza-Perez AI, Kenney S, Boswell J, Hollingshead M, Alley MC, Hose C, Ciolino HP, Yeh G Ch, Trepel JB, Vistica D and Sausville EA: Aryl hydrocarbon receptor activation of aminoflavone (NSC 686288): basis of selective toxicity for MCF-7 breast cancer cells. Mol Cancer Ther 3: 715-725, 2004.

6. Loaiza-Perez AI, Kenney S, Boswell J, Hollingshead M, Hose C, Lineham WM, Worrell R, Rubinstein L, Sausville EA and Vistica DT: Sensitivity of renal cell carcinoma to aminoflavone: role of CYP1A1. J Urol 171: 1688-1697, 2004.

7. Meng LH, Shankavaram U, Chen Ch, Agama K, Fu HQ, Gonzalez FJ, Weinstein J and Pommier Y: Activation of aminoflavone (NSC 686288) by a sulfotransferase is required for the antiproliferative effect of the drug and for induction of histone gamma-H2AX. Cancer Res 66: 9656-9664, 2006.

8. McLean L, Soto U, Agama K, Francis J, Jimenez R, Pommier Y, Sowers L and Brantley E: Aminoflavone induces oxidative DNA damage and reactive oxidative species-mediated apoptosis in breast cancer cells. Int J Cancer 122: 1665-1674, 2008.

9. Meng LH, Kohlhagen G, Liao ZY, Antony S, Sausville E and Pommier Y: DNA-protein cross-links and replication-dependent histone $\mathrm{H} 2 \mathrm{AX}$ phosphorylation induced by aminoflavone (NSC 686288), a novel anticancer agent active against human breast cancer cells. Cancer Res 65: 5337-5343, 2005.

10. Shelton P, Sausville E, Nakanishi T, et al: Breast cancer cells resistant to anti-hormone treatments retain sensitivity to aminoflavone (NSC 686288). In: Proceedings of the AACRNCI-EORTC International Conference. Molecular Targets and Cancer Therapeutics, San Francisco, CA, 2007.

11. Berghard A, Gradin K, Pongratz I, Whitelaw M and Poellinger L: Cross-coupling of signal transduction pathways: the dioxin receptor mediates induction of cytochrome P-450IA1 expression via a protein kinase C-dependent mechanism. Mol Cell Biol 13: 677-689, 1993. 
12. Nordeen SK: Luciferase reporter gene vectors for analysis of promoter and enhancers. Biotechniques 6: 454-457, 1988.

13. Meng LH, Zhang H, Hayward L, Takemura H, Shao RG and Pommier Y: Tetrandine induces early G1 arrest in human colon carcinoma by down-regulating the activity and inducing the degradation of G1-S-specific cyclin-dependent kinases and by inducing p53 and p21Cip1. Cancer Res 64: 9086-9092, 2004.

14. Meng LH, Kohn KW and Pommier Y: Dose-response transition from cell cycle arrest to apoptosis with selective degradation of $\mathrm{Mdm} 2$ and $\mathrm{p} 21^{\mathrm{WAF} / \mathrm{CIPl}}$ in response to the novel anticancer agent, aminoflavone (NSC 686288). Oncogene 26: 4806-4816, 2007.

15. Steele RJC and Lane DP: p53 in cancer: a paradigm for modern management of cancer. Surgeon 3: 197-205, 2005.
16. Dutcher JP, de Souza P, McDermott D, Figlin RA, Berkenblit A, Thiele A, Krygowski M, Strahs A, Feingold J and Hudes G: Effect of temsirolimus versus interferon-alpha on outcome of patients with advanced renal cell carcinoma of different tumor histologies. Med Oncol 26: 202-209, 2009.

17. Terzuoli E, Puppo M, Rapisarda A, Uranchimeg B, Cao L, Burger A, Ziche M and Melillo G: Aminoflavone, a ligand of the aryl hydrocarbon receptor, inhibits HIF-1 $\alpha$ expression in an AhR-independent fashion. Cancer Res 70: 6837-6847, 2010.

18. Turcotte S, Chan D, Sutphin P, Hay M, Denny W and Giaccia A: A molecule targeting VHL-deficient renal cell carcinoma that induces autophagy. Cancer Cell 14: 90-102, 2008. 